Association for Information Systems

AIS Electronic Library (AISeL)

\title{
ArgueBot: A Conversational Agent for Adaptive Argumentation Feedback
}

Thiemo Wambsganss

Universität St.Gallen

Sebastian Guggisberg

Universität St.Gallen

Matthias Soellner

Universität Kassel

Follow this and additional works at: https://aisel.aisnet.org/wi2021

Wambsganss, Thiemo; Guggisberg, Sebastian; and Soellner, Matthias, "ArgueBot: A Conversational Agent for Adaptive Argumentation Feedback" (2021). Wirtschaftsinformatik 2021 Proceedings. 2.

https://aisel.aisnet.org/wi2021/PHuman/Track11/2

This material is brought to you by the Wirtschaftsinformatik at AIS Electronic Library (AISeL). It has been accepted for inclusion in Wirtschaftsinformatik 2021 Proceedings by an authorized administrator of AIS Electronic Library (AISeL). For more information, please contact elibrary@aisnet.org. 


\title{
ArgueBot: A Conversational Agent for Adaptive Argumentation Feedback
}

\author{
Thiemo Wambsganss ${ }^{1}$, Sebastian Guggisberg ${ }^{1}$, and Matthias Söllner ${ }^{2}$ \\ ${ }^{1}$ University of St.Gallen (HSG), Institute of Information Management, St.Gallen, Switzerland \\ thiemo.wambsganss@unisg.ch, sebastian.guggisberg@student.unisg.ch \\ ${ }^{2}$ University of Kassel, Information Systems and Systems Engineering, Kassel, Germany, \\ soellner@uni-kassel.de
}

\begin{abstract}
By combining recent advances in Natural Language Processing and Conversational Agent (CAs), we suggest a new form of human-computer interaction for individuals to receive formative feedback on their argumentation to help them to foster their logical reasoning skills. Hence, we introduce ArgueBot, a conversational agent, that provides adaptive feedback on students' logical argumentation. We, therefore, 1) leveraged a corpus of argumentative student-written peer-reviews in German, 2) trained, tuned, and benchmarked a model that identifies claims, premises and non-argumentative sections of a given text, and 3) built a conversational feedback tool. We evaluated ArgueBot in a proof-of-concept evaluation with students. The evaluation results regarding technology acceptance, the performance of our trained model, and the qualitative feedback indicate the potential of leveraging recent advances in Natural Language Processing for new human-computer interaction use cases for scalable educational feedback.
\end{abstract}

Keywords: Argumentation Learning, Argumentation Mining, Pedagogical Conversational Agents

\section{$1 \quad$ Introduction}

In today's world most information is readily available and the importance of the ability to reproduce information is decreasing. This results in a shift of job profiles towards interdisciplinary, ambiguous and creative tasks [1]. Thus, educational institutions are asked to evolve in their curricula when it comes to the compositions of skills and knowledge conveyed [2]. Especially teaching higher order thinking skills to students, such as critical thinking, collaboration or problem-solving, have become more important [3]. This has already been recognized by the Organization for Economic Cooperation and Development (OECD), which included these skills as a major element of their Learning Framework 2030 [4]. One subclass represents the skill of arguing in a structured, reflective and well-formed way [5]. Argumentation is not only an essential part of our daily communication and thinking but also contributes significantly to the competencies of communication, collaboration and problem-solving [6]. Starting with studies from Aristoteles, the ability to form convincing arguments is recognized as the foundation for persuading an audience of novel ideas and plays a major role in strategic 
decision-making and analyzing different standpoints especially in regard to managing digitally enabled organizations. To develop skills such as argumentation, it is of great importance for the individual to receive continuous feedback throughout their learning journey, also called formative feedback $[7,8]$ However, universities, face the challenge of providing individual learning conditions, since every student would need a personal tutor to have an optimal learning environment to learn how to argue [9]. However, this is naturally hindered due to traditional large-scale lectures or due to the growing field of distance learning scenarios such as massive open online courses (MOOCs) [10].

A possible solution avenue lies in leveraging Natural Language Processing (NLP) and Machine Learning (ML) to provide students with adaptive and ongoing feedback, e.g., on texts and instant messages by a Conversational Agent (CA) [11] and thus provide them access to formative argumentation feedback. CAs are software programs that communicate with users through natural language interaction interfaces $[12,13]$. The successful application of CAs to meet individual needs of learners and to increase their learning outcomes has been demonstrated for learning various skills such as problem-solving skills [14], programming skills [15], mathematical skills [16] as well as for learning factual knowledge [17], and also offers potential for training argumentation skills. A possible solution to provide adaptive support of argumentation could be the utilization by argumentation mining, a proven approach to identify and classify argumentation in texts. The potential of argumentation mining has been investigated in different research domains, such as automated skill learning support for students [18-20], accessing argumentation flows in legal texts [21], better understanding of customer opinions in user-generated comments [22], or fact-checking and de-opinionizing of news [23]. Hence, we suggest that the advantages of argumentation mining could be leveraged to design a new form of human-computer interaction for individual to receive scalable formative argumentation feedback [24]. In fact, Lippi and Torroni (2016) [25] and Chernodub et al. (2019) [26] designed static argumentation web interfaces that can be used to automatically identify and classify argumentation components from English input texts. However, these tools fall short to provide an educational embedding, a user-friendly form of human-computer interaction, e.g., through a conversational interface, and lack application for German content, since they were trained on English corpora. Therefore, we aim to close this gap by designing a CA that can be used by individual learners to receive formative argumentation feedback, e.g., while writing an argumentative text. Overall, we aim to contribute to research and practice by answering the following research question:

RQ: How do students perceive a conversational agent which provides adaptive argumentation feedback on a given text based on Argumentation Mining?

To tackle the research question, we develop a CA in Slack (Slack bot) called ArgueBot (short for Argumentation Bot), which provides students feedback on the logical argumentation of given texts on the baseline of existing theory (cognitive

\footnotetext{
${ }^{1}$ https://slack.com/
} 
dissonance based on [27]). We believe cognitive dissonance theory could explain why formative feedback on an individual's argumentation will motivate the individual to learn how to argue. With adaptive formative feedback, we implicate a CA which provides individual and real-time feedback to individuals on a given text.

We follow the Cross Industry Standard Process for Data Mining (CRISP-DM) Model [28] to build a novel model that identifies argumentation flaws in texts. For training our argumentative feedback model, we leverage the German business model feedback corpus of [29]. We develop a novel modelling approach to identify argumentative structures and build a CA in Slack, which provides individuals with formative argumentation feedback based on given textual messages. To answer our research question, we evaluate ArgueBot in a first proof-of-concept evaluation, where we ask students to write an argumentative text and use our CA to receive feedback on the argumentation structure. The measured technology acceptance, the positive qualitative feedback and the strong performance of our model compared to state-of the art approaches suggest using new forms of conversational human-computer interaction based on NLP to provide students with argumentation support in different scenarios.

The remainder of the paper is structured as follows: First, we provide the necessary conceptual background on argumentation learning, argumentation mining and CAs based on a systematic literature review [1]. Next, we present our CRISP-DM methodology in section three and explain the implementation and evaluation of our model and ArgueBot in section four. Finally, we present and evaluate our results, followed by a discussion about the limitations and contributions of our study.

\section{Conceptual and Theoretical Background}

\subsection{Adaptive Argumentation Learning}

As Kuhn (1992) [6] states, the skill to argue is of great significance not only for professional purposes like communication, collaboration and for solving difficult problems but also for most of our daily life: "It is in argument that we are likely to find the most significant way in which higher order thinking and reasoning figure in the lives of most people. Thinking as argument is implicated in all of the beliefs people hold, the judgments they make, and the conclusions they come to; it arises every time a significant decision must be made. Hence, argumentative thinking lies at the heart of what we should be concerned about in examining how, and how well, people think" ([6], pp. 156-157). However, teaching argumentation is limited. [30] identified three major causes for that: "teachers lack the pedagogical skills to foster argumentation in the classroom, so there exists a lack of opportunities to practice argumentation; external pressures to cover material leaving no time for skill development; and deficient prior knowledge on the part of learners". Therefore, many authors have claimed that fostering argumentation skills should be assigned a more central role in our educational system [31,32]. Adaptive support approaches for argumentation learning (e.g., 15, 3032) describe a rather new field of argumentation learning supported by IT-based systems. The aim is to provide pedagogical feedback on a learner's action and solutions, 
hints and recommendations to encourage and guide future activities in the writing processes or automated evaluation to indicate whether an argument is syntactically and semantically correct. However, the combination of NLP, ML and pedagogically evaluated formative feedback in a student's learner journey is merely investigated due to high complexity. As Scheuer (2015) identifies, "rigorous empirical research with respect to adaptation strategies is almost absent; a broad and solid theoretical underpinning, or theory of adaptation for collaborative and argumentative learning is still lacking" [36]. Therefore, we aim to address this research gap and design an easyto-use argumentation learning tool based on a conversational human-computer interaction design. Thus, we built on the application of recent developments in NLP and ML, in which argumentation mining has been a proven approach to identify and analyze argumentative structures of a given text in real time [19, 29, 37, 38].

\subsection{Argumentation Mining}

The foundation of argumentation mining is argumentation theory. Argumentation theory is about analyzing the structure and the connection between arguments. One of the most prominent argumentation models is the Toulmin model [39]. Toulmin's model asserts that a "good" argument involves a logical structure built on ground, claim and warrant, whereas the grounds are the evidence used to prove a claim. Walton et al. (2008) [40] developed the so-called "argumentation schemes" that use the Toulmin's type of reasoning. It is commonly considered that "Claim", "Premise", and "Warrant" are the main components of every argument, and the rest are supporting sub-argument parts that may or may not exist in an argument. Argumentation mining itself aims to identify these components of an argumentation model with NLP and ML. It falls under the category of computational argumentation, which encompasses a variety of tasks. These tasks include identifying the argumentation style [41], in which arguments are classified as "factual" or "emotional" in order to understand the characteristics better, identifying the reasoning behind the stance of the author by creating a classifier using the stance classification [42], identifying arguments to be used as summarization pointers [43] or ranking arguments according to how convincing they are using a joint model with one deep learning module in it [44]. Following [45], the most related subtasks of argumentation mining can be summed up as:

- Argument Identification, which is concerned with identifying the argumentative parts in raw text and setting up its boundaries versus a nonargumentative text.

- Argument component classification, which is the subtask of finding out what the primary purpose is to classify the components of the argument structure. Classifying an argumentative text into claims or premises is one popular way of tackling the target of this subtask.

- Argumentative discourse analysis, during this subtask, the researcher tries to identify the discourse relations between the various components existing in the argument. A typical example of this subtask is the identification of whether a support or an attack relationship exists between the claim and the premise. 
In our study we are focusing on the challenges of argument identification and argument component classification since these are usually the first two steps of an argumentation mining architecture and, thus, the foundation of every argumentation mining application. The potential of argumentation mining has been investigated in different research domains. However, it has merely been leveraged to build a conversational learning tool to provide individuals with formative and adaptive argumentation feedback on a commonly available communication platform such as Slack $[20,37]$.

\subsection{Adaptive Argumentation Feedback Tools and Conversational Agents}

Although argumentation mining is a growing field of research and many studies have been conducted, only very few practical tools exist that provide individuals - nonprogrammers - with access to this technology. In a systematic literature review, we found only two tools available that provide individuals with access to argumentation mining. Lippi and Torroni (2016) [25] developed the first online argumentation mining tool that was made available for a broad audience. Their tool, MARGOT, is available as a web application and processes a text that is input in the corresponding editor field. After processing and analyzing the text, the results are displayed on the user interface. Claims are displayed in bold font, whereas premises are displayed in italic style [25]. The second and most recent tool that provides individuals with access to argumentation mining was published by [26]. Similar to MARGOT, in their system called TARGER a user can analyze the argumentative structure of an input text. The results are then presented below the input, whereas claims are highlighted in red and premises are marked in green. However, both approaches are only available in English, not designed from an educational perspective and thus not necessarily easy-to-use and easy-to-access for students in their learning journey, since a user would always have to open the website, select a certain model and then copy his or her text into the input field. Therefore, we suggest building a novel conversational interface for leveraging argumentation mining for argumentation feedback for students.

CAs are software programs that are designed to communicate with users through natural language interaction interfaces [12,13]. In today's world, conversational agents, such as Amazon's Alexa, Google's Assistant or Apple's Siri, are ubiquitous, with their popularity steadily growing over the past few years $[46,47]$. They are implemented in various areas, such as customer service [11], collaboration [48], counselling [49] or education (e.g., [50, 51]). Hobert and Wolff (2019) [52] define CAs used in education as a special form of learning application that interacts with learners individually. We believe that a CA can offer new forms of providing argumentative feedback to students through a conversation interface. Therefore, we aim to tackle the challenge of adaptive argumentation support to individuals by building a novel model based on argumentation mining and prototype a conversational interface on an open platform such as Slack. 


\subsection{Cognitive Dissonance Theory}

We built our research project on cognitive dissonance theory. We believe that this theory might supports our underlying hypothesis that individual and personal feedback on a individuals' argumentation motivates the individual to improve her skill level. Cognitive dissonance refers to the unsatisfying feeling that occurs when there is a conflict between one's existing knowledge and contradicting presented information [27]. This uncomfortable internal state results in a high motivation to solve this inconsistency. According to Festinger's theory, an individual experiencing this dissonance has three possible ways to resolve it: change the behavior, change the belief or rationalize the behavior. Especially for students in a learning process, dissonance is a highly motivating factor to gain and acquire knowledge to actively resolve the dissonance [53]. It can be an initial trigger for a student's learning process and thus the construing of new knowledge structures [54]. However, the right portion of cognitive dissonance is very important for the motivation to solve it. According to Festinger, individuals might not be motivated enough to resolve it if the dissonance is too obvious, whereas a high level of dissonance might lead to frustration. Therefore, we believe that the right level of feedback on a student's skill through a conversational interface on an open communication platform, could lead to cognitive dissonance and thus to motivation to change the behavior, belief or knowledge to learn how to argue [55].

\section{$3 \quad$ Research Methodology}

To answer our research question, we develop an artifact following the Cross Industry Standard Process for Data Mining (CRISP-DM) Model, which is illustrated in Figure 1 [28]. The model describes a standardized approach for Data Mining problems from a practical point of view, followed by the data understanding, the data preparation, and the data modelling.

Our approach is divided into five iterative stages. In the first stage, we analyzed the current state of argumentation learning and argumentation mining achievements in literature based on a systematic literature review [1]. Second, we investigated different corpora and their results at the current state in argument classification across multiple domains. We built on the corpus of [29] since it fulfills our requirements of a large data set, a rigorous annotation study and has been successfully used to provide students adaptive argumentation feedback [19]. Third and fourth, we built a model using NLP and Deep Learning (DL) algorithms to classify a text piece as a claim, premise or nonargumentative following the model of $[38,39,56]$. We iteratively evaluated the performance of our model and revised it based on various performance metrics such as the f1-score. In a fifth step, the model is deployed as the back-end algorithm of our conversational agent ArgueBot using the Slack communication platform. We chose Slack since it is a team communication platform widely used with a strong user growth [57]. Moreover, Slack offers an easy-to-use API for building CAs. We believe that a Slack bot might lower the barrier for students to use such a feedback tool in their daily learning journey. Finally, to we evaluate ArgueBot in a proof-of-concept evaluation, following the evaluation patterns of artefacts of Sonnenberg and vom Brocke (2012). 


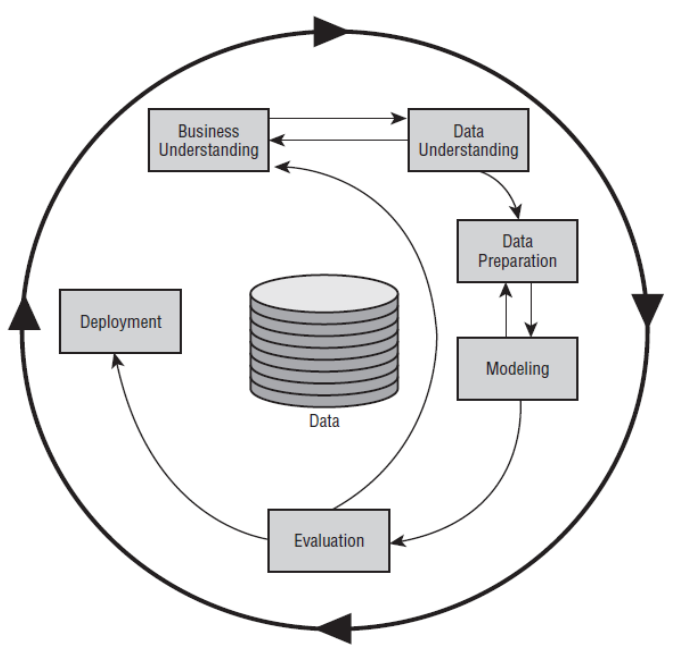

Figure 1. Cross Industry Standard Process for Data Mining (CRISP-DM) [28]

Our approach is developed using the programming language Python 3.7 for Machine Learning (ML) applications, since it is widely known, easy to use and supports major libraries for NLP and ML tasks. For Deep Learning, TensorFlow and its integrated Keras [59] are called. Additionally, the framework FARM by deepset ${ }^{2}$ was used.

\section{Implementation and Evaluation of ArgueBot}

To answer our research question, we aim to build a CA that provides adaptive feedback on the argumentation structure of a given text input by identifying claims, premises and non-argumentative sections. In order to accomplish this, we propose to train a model on a transfer learning approach based on Deep Bidirectional Transformers for Language Understanding (BERT) as seen in [60]. The first phase of CRISP-DM, business understanding, is explained in the introduction and the theoretical background of this work.

\subsection{Data Understanding and Data Preparation}

For training our argumentative feedback model, we leverage the German business model feedback corpus of [29] since it provides a) a large data base of 1000 argumentation annotated student texts in German language, is b) annotated based on the argumentation theory of Toulmin [39] and c) provides a rigorous annotation study with a moderate agreement. We split the corpus' texts into tokens and assigned the corresponding label. In our case, the label includes two parts. The first part represents the IOB-encoding following [61]. The IOB-encoding indicates whether a token is the

\footnotetext{
${ }^{2}$ https://github.com/deepset-ai/FARM
} 
beginning of an argument component ("B" = beginning), is covered by an argument component ("I" = inside) or not included in any argument component ("O"= outside). Additionally, we include the specific argument type in the label, which results in labels such as "B-Premise" (standing for a token being the beginning of a premise) or " $I$ Claim" (standing for a token being inside a claim). Since several authors reached satisfying results with bidirectional Long-Short-Term-Memory-Conditional-RandomFields classifiers (BiLSTM-CRF), we started with such a modelling architecture [26, 62]. To prepare the data accordingly, the tokens are replaced by their index in the corresponding word embeddings vocabulary (GloVe) [63]. However, since we did not receive sufficient accuracy in the further refinement of our model, the architecture was switched to the Bidirectional Encoder Representations from Transformers (BERT) proposed by Devlin et al. (2018) [60] in a second modelling cycle (see section 4.2). Therefore, the tokens are further split into word pieces to fulfill the preparation requirements for BERT. This also requires providing additional labels for the word piece endings. Finally, the data are transformed to PyTorch Tensors ${ }^{3}$, which represent multi-dimensional matrices containing a single data type, in order to match the model's input requirements for the used framework. The special preprocessing for BERT was conducted by utilizing the tokenizer and processor provided by the FARM framework from deepset.

\subsection{Iterative Modelling: Identifying Claims and Premises}

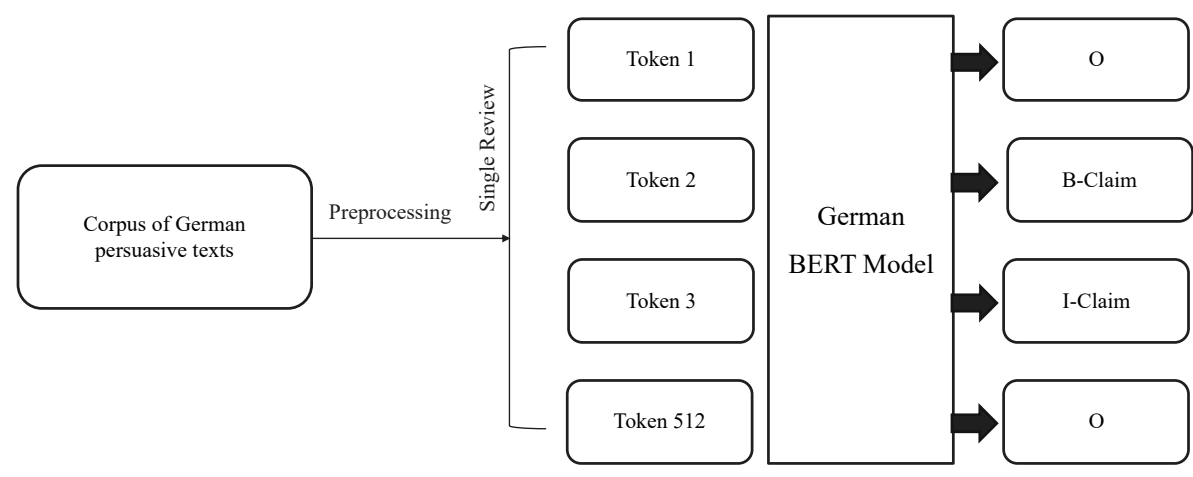

Figure 2. Overview of the model architecture based on BERT [60]

The goal of our model is to provide accurate predictions to identify and classify argument components that can be used for an automated argument feedback system. We split the data into $70 \%$ training, $20 \%$ validation and $10 \%$ test data. We iteratively developed our model in two cycles. In the first cycle, the current state-of-the-art model, a bidirectional LSTM-CRF classifier with GloVe embeddings input, was created following the approach for persuasive essays of [62]. However, since we only reached an unsatisfying f1-score of 57 percent, we decided to follow a more novel transfer

\footnotetext{
${ }^{3}$ https://pytorch.org/docs/stable/tensors.html
} 
learning approach in the second modelling interaction. In fact, we decided to change the architecture of our model to the Bidirectional Encoder Representations from Transformers (BERT) proposed by Devlin et al. (2018) [60]. BERT has been successfully used to model argumentations across different domains [38]. A German BERT model is available through the framework FARM. For the proposed architecture, the inputs and outputs are adapted to the sequence classification task of argument component identification and classification. The last hidden layer is a Recurrent Neural Network with 512 nodes that takes the BERT output and learns to feed into a sigmoid layer that classifies each token according to the predicted label. Figure 2 illustrates the basic architecture of our model following BERT.

The proposed model was finetuned in several iterations and the best performing set of hyperparameters included a learning rate of $5 e^{-5}$, a warmup and embedding dropout probability of 0.1 and 0.15 respectively. As presented in Table 2, BERT clearly outperforms other state-of-the-art model architectures for argumentation mining for persuasive essays (e.g., [62]). Whereas the bidirectional LSTM-CRF classifier achieved a macro f1-score of 0.57 , the German BERT model performed about $28 \%$ better, reaching a macro f1-score of 0.73 on the token classification task on the German student written business model review corpus.

Table 2. Overview of overall performance of the BiLSTM-CRF and BERT

\begin{tabular}{|c|c|c|c|}
\hline Model & Precision & Recall & F1-Score \\
\hline BiLSTM-CRF & 0.60 & 0.55 & 0.57 \\
\hline BERT & 0.74 & 0.72 & 0.73 \\
\hline
\end{tabular}

After a set of iterative refinements, we achieved satisfying results for the classification of argumentative components of the texts using the BERT model of deepset. The results are stated in Table 3 for the different labels.

Table 3. Overview of results of BERT for the classification of argumentative components

\begin{tabular}{|c|c|c|c|}
\hline Label & Precision & Recall & F1-Score \\
\hline B-Claim & 0.66 & 0.74 & 0.69 \\
\hline I-Claim & 0.72 & 0.62 & 0.66 \\
\hline B-Premise & 0.66 & 0.75 & 0.70 \\
\hline I-Premise & 0.75 & 0.68 & 0.72 \\
\hline O & 0.91 & 0.82 & 0.86 \\
\hline
\end{tabular}

\subsection{Deployment: Building ArgueBot - a CA for Adaptive Argumentation Feedback}

In order to contribute to our research question, the next step incorporated to deploy our trained model in a conversational interface so that students can receive formative feedback on their argumentation structure, e.g., when writing an argumentative essay. Therefore, the trained model was exported and implemented in the back-end of our CA ArgueBot which identifies and classifies argument components and provides feedback 
on the argumentative structure. We chose Slack as a platform for ArgueBot since it is a team communication platform widely used by many large organizations. We believe that a CA in Slack (Slack bot) might lower the barrier for using such a feedback system in daily learning activities. A screenshot of our final CA in Slack is illustrated in Figure 3.

The functionality and usability are kept rather simple so that students can access our CA without any pre-knowledge or onboarding. The user can enter a message and send it to ArgueBot, e.g., an argumentative essay or an important email (see Figure 3: 1). This message is then sent to our trained model. Claims, premises and nonargumentative tokens are being classified and sent back to the frontend. Following [25], claims are then displayed in bold font, whereas premises are displayed in italic style (see Figure 3: 2). Besides, ArgueBot is providing a short summarizing feedback based on the number of premises and claims in the message (see Figure 3:3). For example, if the message contains less than two premises or contains more claims than premises, the user receives a corresponding feedback indicating that the argumentation is not sufficient. We believe that this individual and personal feedback on an individual's argumentation motivates the individual to improve her skill level.

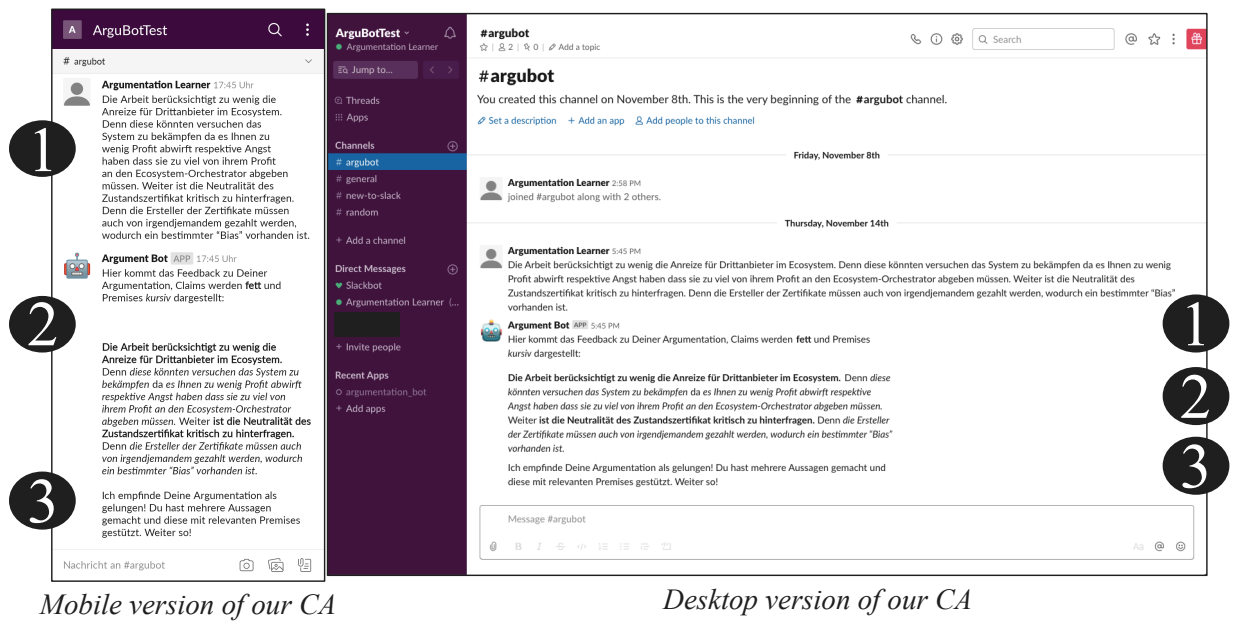

Figure 3. Screenshots of our CA ArgueBot providing argumentative feedback to a student

\subsection{Proof-of-Concept Evaluation of ArgueBot}

In order to answer our research question and evaluate the feasibility of ArgueBot we performed a proof-of-concept evaluation following the evaluation patterns of technical artifacts of [58]. Hence, our aim was to investigate how do students perceive a conversational agent which provides adaptive argumentation feedback on given texts. Therefore, we performed a qualitative and a quantitative evaluation. We designed a laboratory experiment, where we asked students to write an argumentative text message, e.g., a statement about a business model. The participants were told that the 
test message should contain at least three sentences (around 50 words). We asked the participants to use ArgueBot to receive feedback on the argumentation structure of their text and revise it accordingly, if wished. After that the participants were asked to conduct a post-survey. We captured the perceived usefulness, intention to use and ease of use following the technology acceptance model of $[64,65]$. Exemplary items for the three constructs are: "Imagine the feedback tool would be available in your daily working life, I am encouraged to use it.", "I would find the feedback tool useful for receiving feedback on my argumentation.", "Using the feedback tool would allow me to write more argumentative messages." or "The feedback tool is easy to use." All these items were measured with a 7-point Likert scale (1: strongly disagree to 7: strongly agree, with 4 being a neutral statement). In addition, we collected demographic information, such as age, gender and occupation. Finally, the participants were asked to provide qualitative feedback regarding the strengths, weaknesses and suggestions for useful additional features.

In total, ten users participated in our experiment; eight male and two females, who were between the ages of 22 and 25. Four participants were software development trainees working in a software company, six participants were business students on master level. The quantitative evaluation of the results showed that the $\mathrm{CA}$ is considered to be easy to use with an overall score of 6.33. The intention to use and the perceived usefulness were rated similar positively, with total scores of 5.5 and 5.63 respectively. A positive technology acceptance is especially important for learning tools to ensure students are perceiving the usage of the tool as helpful, useful and easy to interact. This will foster motivation and engagement to use the learning application. The perceived usefulness and intention to use provides promising results to use this tool as a feedback application in different learning settings.

Moreover, we performed a qualitative evaluation by asking the participants to provide a more detailed feedback on what they particularly liked or disliked about the tool. We clustered the answers to form a more concise feedback. Based on that, it seems that the short adaptive feedback on the argumentation structure is highly appreciated. The participants mentioned several things about the conversational interaction, such as about the format of the feedback ("The formatting of the feedback is very clear"), the reaction time of the CA ("The fast reaction of the system (feedback time) is pleasant"), the differentiation of claims and premises ("I like the division of my statement into Claim and Premise") or the overall feedback system ("The tool really helps to build up a meaningful argumentation structure, it recognized my rather bad argumentation immediately"). However, three of ten participants mentioned that the tool did not find the right argument components. Further, one participant also criticized the speed and the representation of the feedback, since the analysis takes too long and especially the premises (italic) are not clearly highlighted. Besides, several suggestions have been made for further development of such a feedback system, such as clear suggestions to improve the argumentation, spelling checks, scoring or a more detailed description of claims and premises. 


\section{Discussion and Conclusion}

In our paper, we aimed to investigate if a conversational agent based on a novel modelling approach might provide a new form of human-computer interaction for formative argumentation skill feedback. To answer our research question, we develop a new argumentation classification pipeline based on the current state of transfer learning to build a CA that provides students with formative feedback on their argumentation, e.g., when writing an argumentative essay. We built on an existing corpus of argumentation annotated student texts in German to develop a novel modelling approach to identify argumentative structures and build a CA in Slack, which can be used by students in their daily learning activities to receive adaptive and ongoing feedback independent of an instructor, time and location. To evaluate how do students perceive a conversational agent which provides adaptive argumentation feedback on given text, we performed a first proof-of-concept evaluation, where we asked participants students to write an argumentative text and to use our CA for receiving adaptive feedback. Our results indicate that students would intent to use a conversational argumentation feedback tool. Moreover, the measured perceived usefulness and perceived ease of use provides evidence that this new form of humancomputer interaction might help to leverage recent advantages in NLP to provide students with writing support through a conversational interface. In order to successfully use a learning tool in a real-world scenario, positive technology acceptance is very important to ensure students perceive the usage of the tool as helpful, useful and easy to interact with. This will foster motivation and engagement to use the learning application.

We build our research on cognitive dissonance theory [27]. We argue that a learning tool for argumentation skills (and possibly also meta cognition skills) like our CA increases the motivation of students to learn how to apply the certain skills, for example, learn how to argue, and thus improve the learning outcome. For example, our ArgueBot which provides instant and individual feedback should increase the individual's motivation to resolve dissonance and therefore construct new knowledge. This goes along with other studies on adaptive argumentation support in the literature of HCI research (e.g., [19, 55]).

Thereby, our study contributes to two different research areas in information systems: first, we contribute to new forms of human-computer interaction in adaptive education, suggesting a use case to employ a conversatioanl interaction with potential benefits for educational institutions and organizations to foster adaptive and on-going skill feedback. We show how an exemplary case, based on a CA and a novel NLP model, can be leveraged to provide individual support and feedback to enable students to receive feedback on a certain skill. Second, we contribute to the field of digital learning innovations, by embedding a recent technology from NLP and ML to help students to learn how to argue independent of a human, time and location.

Nevertheless, our study faces some limitations. First, our evaluation displays a proof-of-concept evaluation about the technology acceptance of our CA. We did not evaluate the influence of argumentation feedback through a CA on the actual argumentation skills. However, we believe cognitive dissonance theory might explain 
how adaptive feedback leads to the motivation to learn. Moreover, our evaluation is based on a rather small sample size. More participants in an evaluation are needed to strengthen the findings. Therefore, we call for future research to further investigate the potential of new conversation-based forms of human-computer interaction for adaptive skill learning. Empirical studies are needed to investigate the effects of new interactive learning tools on students' skill level. Moreover, we did not investigate and evaluate specific design cues of a CA for adaptive argumentation feedback, since we wanted to provide a proof-of-concept study rather than a design science research approach. A more user-centered design approach, however, would be necessary to further investigate design parameters and design principles for adaptive conversation-based learning tools.

All in all, we contribute to a new, unified approach for adaptive argumentative support of students by showing an exemplary use case for argumentation skill learning. Researcher can build on this to investigate new HCI use cases for other skills in which adaptive feedback might be necessary for formative learning in large-scale or distance learning scenarios. With further advances of NLP and ML, we hope our work will attract researchers to design more intelligent tutoring systems for other learning scenarios or metacognition skills and thus contribute to the OECD Learning framework 2030 towards a metacognition-skill-based education.

\section{References}

1. vom Brocke, J., Simons, A., Riemer, K., Niehaves, B., Plattfaut, R., Cleven, A.: Standing on the shoulders of giants: Challenges and recommendations of literature search in information systems research. Commun. Assoc. Inf. Syst. 37, 205-224 (2015). https://doi.org/10.17705/1 cais.03709.

2. Topi, H.: Using competencies for specifying outcome expectations for degree programs in computing: Lessons learned from other disciplines. 2018 SIGED Int. Conf. Inf. Syst. Educ. Res. (2018).

3. Fadel, C., Bialik, M., Trilling, B.: Four-dimensional education : the competencies learners need to succeed. (2015).

4. OECD: The Future of Education and Skills - Education 2030, (2018). https://doi.org/201806-15.

5. Toulmin, S.E.: The uses of argument: Updated edition. (2003). https://doi.org/10.1017/CBO9780511840005.

6. Kuhn, D.: Thinking as Argument. Harv. Educ. Rev. 62, 155-179 (1992). https://doi.org/10.17763/haer.62.2.9r424r0113t67011.

7. Black, P., Wiliam, D.: Developing the theory of formative assessment. Educ. Assessment, Eval. Account. 21, 5-31 (2009). https://doi.org/10.1007/s11092-008-9068-5.

8. Hattie, J., Timperley, H.: The Power of Feedback. Rev. Educ. Res. 77, 81-112 (2007). https://doi.org/10.3102/003465430298487.

9. Vygotsky, L.S.: Mind in society: The development of higher psychological processes. Harvard university press (1980).

10. Seaman, J.E., Allen, I.E., Seaman, J.: Higher Education Reports - Babson Survey Research 
Group. (2018).

11. Zierau, N., Wambsganss, T., Janson, A., Schöbel, S., Leimeister, J.M.: The Anatomy of User Experience with Conversational Agents : A Taxonomy and Propositions of Service Clues. Icis 2020. 1-17 (2020).

12. Shawar, B.A., Atwell, E.S.: Using corpora in machine-learning chatbot systems. Int. J. Corpus Linguist. 10, 489-516 (2005). https://doi.org/10.1075/ijcl.10.4.06sha.

13. Rubin, V.L., Chen, Y., Thorimbert, L.M.: Artificially intelligent conversational agents in libraries. Libr. Hi Tech. 28, 496-522 (2010). https://doi.org/10.1108/07378831011096196.

14. Winkler, R., Büchi, C., Söllner, M.: Improving Problem-Solving Skills with Smart Personal Assistants: Insights from a Quasi Field Experiment. Fortieth Int. Conf. Inf. Syst. 1-17 (2019).

15. Hobert, S.: Say hello to 'Coding Tutor'! Design and evaluation of a chatbot-based learning system supporting students to learn to program. 40th Int. Conf. Inf. Syst. ICIS 2019. 1-17 (2019).

16. Cai, W., Grossman, J., Lin, Z., Sheng, H., Tian, J., Wei, Z., Williams, J.J., Goel, S.: MathBot: A Personalized Conversational Agent for Learning Math. (2019). https://doi.org/10.475/123_4.

17. Ruan, S., Jiang, L., Xu, J., Tham, B.J.-K., Qiu, Z., Zhu, Y., Murnane, E.L., Brunskill, E., Landay, J.A.: QuizBot: A Dialogue-based Adaptive Learning System System for Factual Knowledge. 1-13 (2019). https://doi.org/10.1145/3290605.3300587.

18. Stab, C., Gurevych, I.: Identifying Argumentative Discourse Structures in Persuasive Essays. In: Conference on Empirical Methods in Natural Language Processing (EMNLP 2014)(Oct. 2014), Association for Computational Linguistics, p.(to appear). pp. 46-56 (2014).

19. Wambsganss, T., Niklaus, C., Cetto, M., Söllner, M., Leimeister, J.M., Handschuh, S.: AL : An Adaptive Learning Support System for Argumentation Skills. In: ACM CHI Conference on Human Factors in Computing Systems. pp. 1-14 (2020).

20. Wambsganss, T., Söllner, M., Leimeister, J.M.: Design and Evaluation of an Adaptive Dialog-Based Tutoring System for Argumentation Skills. In: International Conference on Information Systems (ICIS). , Hyderabad, India (2020).

21. Moens, M., Boiy, E., Reed, C.: Automatic Detection of Arguments in Legal Texts. Proc. 11th Int. Conf. Artif. Intell. law. ACM. (2007).

22. Boltuži, F., Šnajder, J.: Back Up Your Stance: Recognizing Arguments in Online Discussions. Proc. First Work. Argumentation Min. 1-43 (2014).

23. Dusmanu, M., Cabrio, E., Villata, S.: Argument Mining on Twitter: Arguments, Facts and Sources. Proc. 2017 Conf. Empir. Methods Nat. Lang. Process. 2317-2322 (2018).

24. Lawrence, J., Reed, C.: Argument mining: A survey. Comput. Linguist. 45, 765-818 (2019). https://doi.org/10.1162/COLIa00364.

25. Lippi, M., Torroni, P.: MARGOT: A web server for argumentation mining. Expert Syst. Appl. 65, 292-303 (2016). https://doi.org/10.1016/j.eswa.2016.08.050.

26. Chernodub, A., Oliynyk, O., Heidenreich, P., Bondarenko, A., Hagen, M., Biemann, C., Panchenko, A.: TARGER: Neural Argument Mining at Your Fingertips. 195-200 (2019). https://doi.org/10.18653/v1/p19-3031.

27. Festinger, L.: Cognitive Dissonance. Sci. Am. 207, 93-106 (1962). https://doi.org/10.1038/scientificamerican1062-93.

28. Chapman, P., Clinton, J., Kerber, R., Khabaza, T., Daimlerchrysler, T.R., Shearer, C., Daimlerchrysler, R.W.: Step-by-step data mining guide. SPSS inc. 78, 1-78 (2000). 
29. Wambsganss, T., Niklaus, C., Söllner, M., Handschuh, S., Leimeister, J.M.: A Corpus for Argumentative Writing Support in German. In: 28th International Conference on Computational Linguistics (Coling) (2020).

30. Jonassen, D.H., Kim, B.: Arguing to learn and learning to argue: Design justifications and guidelines. Educ. Technol. Res. Dev. 58, 439-457 (2010). https://doi.org/10.1007/s11423009-9143-8.

31. Driver, R., Newton, P., Osborne, J.: Establishing the norms of scientific argumentation in classrooms. Sci. Educ. 84, 287-312 (2000). https://doi.org/10.1002/(SICI)1098237X(200005)84:3<287::AID-SCE1>3.0.CO;2-A.

32. Kuhn, D.: Education for thinking. Harvard University Press (2005).

33. Pinkwart, N., Ashley, K., Lynch, C., Aleven, V.: Evaluating an Intelligent Tutoring System for Making Legal Arguments with Hypotheticals. IOS Press (2009).

34. Huang, C.J., Chang, S.C., Chen, H.M., Tseng, J.H., Chien, S.Y.: A group intelligence-based asynchronous argumentation learning-assistance platform. Interact. Learn. Environ. 24, 1408-1427 (2016). https://doi.org/10.1080/10494820.2015.1016533.

35. Stab, C., Gurevych, I.: Recognizing Insufficiently Supported Arguments in Argumentative Essays. (2017).

36. Scheuer, O.: Towards adaptive argumentation learning systems. (2015).

37. Lippi, M., Torroni, P.: Argumentation Mining: State of the Art and Emerging Trends. IJCAI Int. Jt. Conf. Artif. Intell., 4207-4211 (2015). https://doi.org/10.1145/2850417.

38. Wambsganss, T., Molyndris, N., Söllner, M.: Unlocking Transfer Learning in Argumentation Mining: A Domain-Independent Modelling Approach. In: 15th International Conference on Wirtschaftsinformatik. , Potsdam, Germany (2020). https://doi.org/10.30844/wi_2020_c9wambsganss.

39. Toulmin, S.E.: Introduction to Reasoning. (1984).

40. Walton, D., Reed, C., Macagno, F.: Argumentation Schemes. Cambridge University Press, Cambridge (2008). https://doi.org/10.1017/CBO9780511802034.

41. Oraby, S., Reed, L., Compton, R.: And That's A Fact: Distinguishing Factual and Emotional Argumentation in Online Dialogue. arXiv Prepr. (2000).

42. Hasan, K.S., Ng, V.: Why are You Taking this Stance ? Identifying and Classifying Reasons in Ideological Debates. Proc. 2014 Conf. Empir. Methods Nat. Lang. Process. 751-762 (2014).

43. Chowanda, A.D., Sanyoto, A.R., Suhartono, D., Setiadi, C.J.: Automatic Debate Text Summarization in Online Debate Forum. Procedia Comput. Sci. 116, 11-19 (2017). https://doi.org/10.1016/j.procs.2017.10.003.

44. Habernal, I., Gurevych, I.: Which argument is more convincing? Analyzing and predicting convincingness of Web arguments using bidirectional LSTM. Proc. 54th Annu. Meet. Assoc. Comput. Linguist. (Volume 1 Long Pap. 1589-1599 (2016).

45. Lippi, M., Torroni, P.: Argumentation Mining : State of the Art and Emerging Trends. ACM Trans. Internet Technol. 16, (2016).

46. Krassmann, A.L., Paz, F.J., Silveira, C., Tarouco, L.M.R., Bercht, M.: Conversational Agents in Distance Education: Comparing Mood States with Students' Perception. Creat. Educ. 09, 1726-1742 (2018). https://doi.org/10.4236/ce.2018.911126.

47. eMarketer: Alexa, Say What?! Voice-Enabled Speaker Usage to Grow Nearly 130\% This Year. (2017). 
48. Elshan, E., Ebel, P.: Let's Team Up: Designing Conversational Agents as Teammates. Int. Conf. Inf. Syst. (2020).

49. Cameron, G., Cameron, D., Megaw, G., Bond, R., Mulvenna, M., O’Neill, S., Armour, C., McTear, M.: Towards a chatbot for digital counselling. HCI 2017 Digit. Make Believe - Proc. 31st Int. BCS Hum. Comput. Interact. Conf. HCI 2017. 2017-July, 1-7 (2017). https://doi.org/10.14236/ewic/HCI2017.24.

50. Wambsganss, T., Winkler, R., Schmid, P., Söllner, M.: Unleashing the Potential of Conversational Agents for Course Evaluations: Empirical Insights from a Comparison with Web Surveys. In: Twenty-Eighth European Conference on Information Systems (ECIS2020). pp. 1-18. , Marrakesh, Morocco (2020).

51. Wambsganss, T., Winkler, R., Söllner, M., Leimeister, J.M.: A Conversational Agent to Improve Response Quality in Course Evaluations. In: ACM CHI Conference on Human Factors in Computing Systems (2020).

52. Hobert, S., Wolff, R.M. Von: Say Hello to Your New Automated Tutor - A Structured Literature Review on Pedagogical Conversational Agents. 14th Int. Conf. Wirtschaftsinformatik, Siegen, Ger. (2019).

53. Elliot, A.J., Devine, P.G.: On the motivational nature of cognitive dissonance: Dissonance as psychological discomfort. J. Pers. Soc. Psychol. 67, 382-394 (1994). https://doi.org/10.1037/0022-3514.67.3.382.

54. Piaget, J., Brown, T., Thampy, K.J.: The Equilibration of Cognitive Structures: The Central Problem of Intellectual Development. Jean Piaget, Terrance Brown, Kishore Julian Thampy. Am. J. Educ. 94, 574-577 (1986). https://doi.org/10.1086/443876.

55. Wambsganss, T., Rietsche, R.: Towards designing an adaptive argumentation learning tool. In: 40th International Conference on Information Systems, ICIS 2019. pp. 1-9. , Munich, Germany (2020).

56. Fromm, H., Wambsganss, T., Söllner, M.: Towards a Taxonomy of Text Mining Features. In: European Conference of Information Systems (ECIS). pp. 1-12 (2019).

57. Statista: Slack - total and paying user count, https://www.statista.com/statistics/652779/worldwide-slack-users-total-vs-paid/, last accessed 2019/11/24.

58. Sonnenberg, C., vom Brocke, J.: Evaluation Patterns for Design Science Research Artefacts. Presented at the October 14 (2012). https://doi.org/10.1007/978-3-642-33681-2_7.

59. Chollet, F.: Keras Documentation, (2015).

60. Devlin, J., Chang, M.-W., Lee, K., Toutanova, K.: BERT: Pre-training of Deep Bidirectional Transformers for Language Understanding. (2018).

61. Ramshaw, L.A., Marcus, M.P.: Text Chunking using Transformation-Based Learning. (1995).

62. Eger, S., Daxenberger, J., Gurevych, I.: Neural End-to-End Learning for Computational Argumentation Mining. In: Proceedings of the 55th Annual Meeting of the Association for Computational Linguistics (Volume 1: Long Papers). pp. 11-22. Association for Computational Linguistics, Stroudsburg, PA, USA (2017). https://doi.org/10.18653/v1/P171002.

63. Pennington, J., Socher, R., Manning, C.D.: GloVe: Global vectors for word representation. In: EMNLP 2014 - 2014 Conference on Empirical Methods in Natural Language Processing, Proceedings of the Conference. pp. 1532-1543. Association for Computational Linguistics 
(ACL) (2014). https://doi.org/10.3115/v1/d14-1162.

64. Venkatesh, V., Bala, H.: Technology acceptance model 3 and a research agenda on interventions. Decis. Sci. 39, 273-315 (2008). https://doi.org/10.1111/j.15405915.2008.00192.x.

65. Venkatesh, V., Morris, M.G., Davis, G.B., Davis, F.D.: User Acceptance of Information Technology: Toward a Unified View. MIS Q. 27, 425-478 (2003). 